\title{
A Critical Evaluation of Dynamical Systems Models of Bipolar Disorder
}

\author{
Abraham Nunes ${ }^{1,2, *}$, Selena Singh ${ }^{3}$, Jared Allman ${ }^{1}$, Suzanna Becker ${ }^{3}$, Abigail Ortiz ${ }^{4,5}$, Thomas \\ Trappenberg $^{2}$, and Martin Alda ${ }^{1}$ \\ ${ }^{1}$ Department of Psychiatry, Dalhousie University, Halifax, Nova Scotia, Canada \\ ${ }^{2}$ Faculty of Computer Science, Dalhousie University, Halifax, Nova Scotia, Canada \\ ${ }^{3}$ Department of Psychology, Neuroscience $\&$ Behaviour, McMaster University, Hamilton, Ontario, Canada \\ ${ }^{4}$ Department of Psychiatry, University of Toronto, Toronto, ON, Canada. \\ ${ }^{5}$ Centre for Addiction \& Mental Health, Toronto, Ontario, Canada \\ *Correspondence: Abraham Nunes (nunes@dal.ca).3083B-5909 Veterans Memorial Lane, Abbie J. Lane Memorial Building, QEII \\ Health Sciences Centre, Halifax, Nova Scotia, B3H 2E2, Canada
}

\begin{abstract}
Bipolar disorder (BD) is a mood disorder involving recurring (hypo)manic and depressive episodes. The inherently temporal nature of $\mathrm{BD}$ has inspired its conceptualization using dynamical systems theory, which is a mathematical framework for understanding systems that evolve over time. In this paper we provide a critical review of dynamical systems models of BD. Owing to heterogeneity of methodologies and experimental designs in computational modeling, we designed a structured approach to guide our review in a fashion that parallels the appraisal of animal models by their Face, Predictive, and Construct Validity. This tool, the Validity Appraisal Guide for Computational Models (VAG-CM) is not an absolute estimate of validity, but rather a guide for more objective appraisal of models in this review. We identified 26 studies published before November 18, 2021 that proposed generative dynamical systems models of time-varying signals in BD. Two raters independently applied the VAG-CM to included studies, obtaining a mean Cohen's $\kappa$ of 0.55 (95\% CI $[0.45,0.64])$ prior to establishing consensus ratings. Consensus VAG-CM ratings revealed three model/study clusters: data-driven models with face validity, theory-driven models with predictive validity, and theory-driven models lacking all forms of validity. We conclude that future models should be developed using a hybrid approach that first operationalizes BD features of interest using empirical data (a data-driven approach), followed by explanations of those features using generative models with components that are homologous to physiological or psychological systems involved in $\mathrm{BD}$ (a theory-driven approach).
\end{abstract}

\section{INTRODUCTION}

Bipolar disorder (BD) is a mood disorder of unknown etiology characterized by episodes of mania, depression, and periods of euthymia [1-5]. Many studies have studied BD during episodes of mania, depression, or euthymia, but fewer have sought to understand the time course of mood transitions and episodicity, despite these properties being hallmarks of $\mathrm{BD}[6]$ and predictive of treatment response [7].

Dynamical systems theory is a mathematical framework for studying systems that evolve over time (reviewed in Appendix A and Lynch [8], Strogatz [9]), which makes it an appealing framework for studying and modeling time-varying aspects of BD. Alternate mechanistic hypotheses can be represented using different model architectures, which can then be compared by fitting the model to empirical data and applying model selection procedures [10]. Use of these methods to study BD dates back more than 20 years [11], but their contributions to the understanding of BD are unclear.

While studies using dynamical systems models in BD may explain known phenomena and generate testable predictions for further research, we currently lack standardized approaches to critically appraise their validity. We therefore sought to review these studies by introducing a set of appraisal criteria that mirror criteria for validating animal models in psychiatry. Using this structured approach, the present review will address the Face, Predictive, and Construct Validity of dynamical systems models of $\mathrm{BD}$, and summarize the insights they have provided about its temporal course and mechanisms. We then highlight conceptual and methodological gaps in this literature, and propose a roadmap for further computational modeling studies of BD.

\section{METHODS}

\subsection{Search Strategy and Evaluation}

The Scopus database was searched from inception until November 18, 2021 using the following search query:

TITLE-ABS-KEY((((affective OR bipolar) AND disorder) OR "manic depression" OR "manic depressive" OR antidepressant) AND ("mood dynamics" OR "mood variability" OR "mood variations" OR circadian OR "biological rhythms") AND (((bistability OR multistability) OR (chaos OR "chaos theory" OR "strange attractor") OR ("lienard oscillators" OR "limit cycle" OR "limit cycle oscillators") OR ("nonlinear dynamics" OR "oscillations" OR "perturbation method" OR "recurrent map" OR "stochastic resonance" OR "winnerless competition") OR ("crisis" OR "critical slowing down" OR intermittency OR "chaotic intermittency")) OR ("computational modelling" OR 
"mathematical modelling" OR "time series analysis" OR "mechacognition" OR "cognitive network"))).

We included studies that have (A) been published in English language peer-reviewed journals, and (B) proposed generative dynamical systems models of mood, activation, circadian rhythm fluctuation, or other time series signals in humans, with respect to $\mathrm{BD}$. We focused on generative models because they do not simply describe some phenomenon, but can also produce it through simulation. Reference lists of included manuscripts were subsequently searched for papers that may have been missed by our initial search. We also searched lists of papers that cited the included studies for further papers that may have been missed.

\subsection{Evaluation of Model Quality}

To evaluate computational models, we devised a set of criteria analogous to those used for validating animal models of neuropsychiatric disorders [12]. These criteria can be broken down into checklists concerning face validity, predictive validity, and construct validity, respectively (Figure 1). A full copy of this Validity Appraisal Guide for Computational Models (VAG-CM) is included Appendix B.

\subsubsection{Validity Appraisal Guide for Computational Models (VAG-CM)}

The VAG-CM is not intended as an absolute evaluation of model validity, but rather as a structured approach to appraise BD studies employing computational models. We attempted to design it with sufficient generality to be equally applicable across a wide range of domains and computational model types, such as those of neurons/circuits [13] and computational cognitive models of memory [14]. By adapting an approach and nomenclature familiar to the psychiatric basic science community, we aimed for the VAG-CM to facilitate successful translation and interplay between computational modelers and experimental psychiatric researchers.

The first subscale, concerning face validity, asks whether a real-world phenomenon has been sufficiently (A) characterized, and then (B) explained by a model. In this subscale, we have tried to capture elements suggestive of convergent and divergent validity. Convergent validity is the degree to which a model of some condition can describe multiple features of that condition. Divergent validity requires that the model can explain features of its target condition, while not being able to capture features of some relevant comparator condition.

1. Face validity: The degree to which the model exhibits a range of behaviours similar to that of the condition of interest. In other words, how good is the model at explaining behaviour in the target condition?

(a) The model aims to describe a real-world phenomenon (i.e. a target state/condition vis a vis comparators)

(b) The target state/condition being modelled is identifiable according to observable features

(c) The comparator state/condition being modelled is identifiable according to observable features (d) The model actually explains/predicts the target condition vis a vis the comparator

The second subscale, concerning predictive validity, asks whether simulated interventions result in behavioral changes similar to those of an analogous real world intervention. For example, if model $M_{1}$ captures depression $(D)$, and model $M_{2}$ captures normal mood (euthymia, $E$ ), then one may identify a real world transition resulting in $D \rightarrow E$ mediated by antidepressants or mood-stabilizers. One could then model that medication-mediated transformation as some function that turns $M_{1} \rightarrow M_{2}$ (thereby resulting in a shift from $D \rightarrow E$ in the model output). Such a model would be a candidate for predictive validity, since the form and consequences of the transformation model could be tested empirically.

2. Predictive validity: The degree to which manipulations of the model predict the effects of real world interventions on the target condition of interest.

(a) There are identifiable and meaningful transitions between conditions/states of interest in the realworld phenomenon

(b) Interventions/transitions in the model explain or predict corresponding transitions in the condition/state of interest

The third and final subscale, concerning construct validity, seeks to evaluate the degree to which proposed models are constrained by empirically derived biological or psychological architectures. For instance, a simple autoregressive model or a complex recurrent neural network can be used to capture mood dynamics, as they are sufficiently general to explain most types of time-series data. However, that generality may not provide much causal insight into the biological or psychological mechanisms underlying the mood fluctuations in BD. A model with construct validity, however, would have an architecture corresponding to biological or psychological properties found to be relevant in BD. For example, biophysical network models may allow us to understand the effects of neuronal excitability on circuit level computations [15]. The results from these computational simulations can then be validated by examining results from real world experiments.

3. Construct validity: The degree of homology between the model architecture and mechanisms that are empirically or theoretically deemed to underlie features of the target condition of interest.

(a) There is a real and identifiable or plausible mechanism underlying the target condition/state

(b) The model architecture is homologous to the mechanism of interest, at an appropriate level of abstraction

A model need not satisfy all three criteria to be useful. A model with good face validity and predictive validity may be of clinical utility, and its lack of construct validity may be irrelevant for the intended use. In fact, a model with face validity alone may be of great benefit for characterizing and identifying features of BD. 


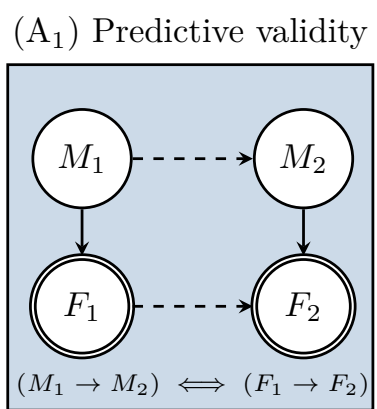

$\left(\mathrm{B}_{1}\right)$ Face validity

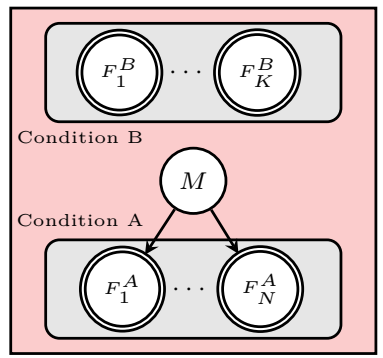

$\left(\mathrm{C}_{1}\right)$ Face validity

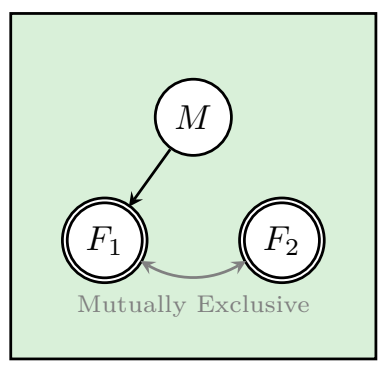

$\left(A_{2}\right)$ No predictive validity $\left(D_{1}\right)$ Construct validity

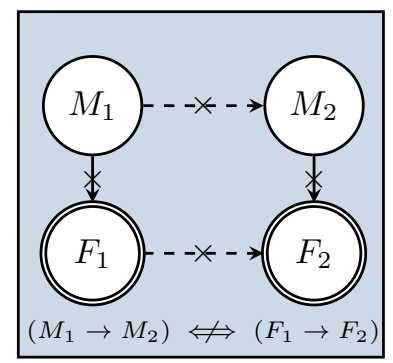

$\left(\mathrm{B}_{2}\right)$ No face validity

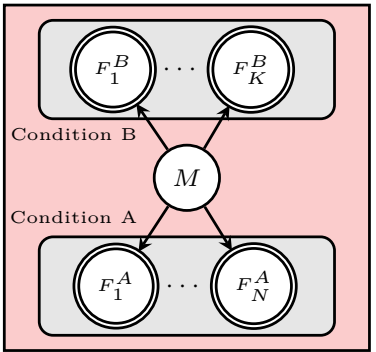

$\left(\mathrm{C}_{2}\right)$ No face validity

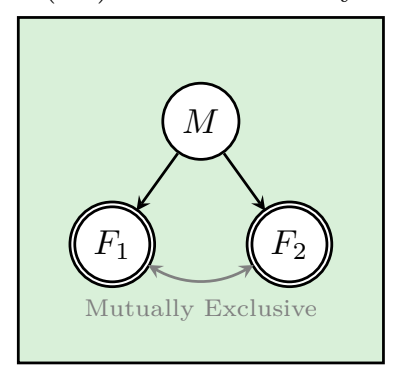

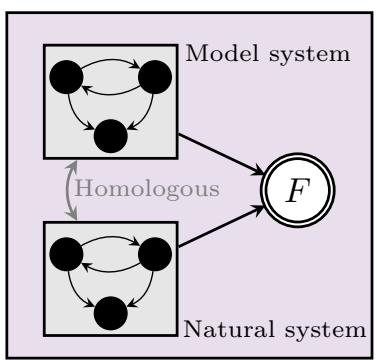

$\left(\mathrm{D}_{2}\right)$ No construct validity

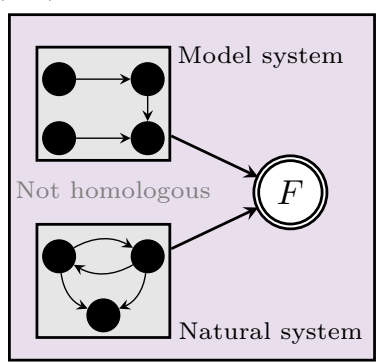

Legend

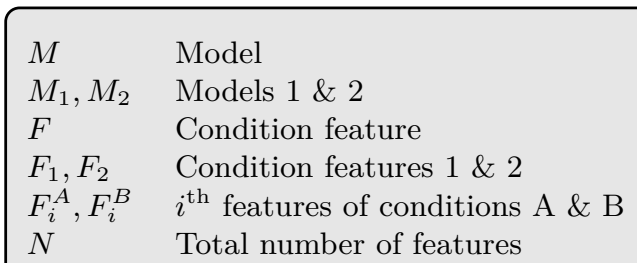

(X) $\begin{aligned} & \text { Empirical } \\ & \text { observation }\end{aligned} \begin{aligned} & \text { Theoretical } \\ & \text { construct }\end{aligned}$

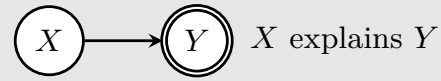

Transformation of $A$ into $B$ explains

transformation of $C$ into $D$, and vice versa:

$$
(A \rightarrow B) \Longleftrightarrow(C \rightarrow D)
$$

Transformation of $A$ into $B$ does not explain transformation of $C$ into $D$, and vice versa:

$$
(A \rightarrow B) \nLeftarrow(C \rightarrow D)
$$

Figure 1: Illustration of validity appraisal guide for computational models of psychiatric disorders. Abbreviations and symbols are shown in the Legend panel. Predictive Validity (Panels $\mathrm{A}_{1}$ and $\mathrm{A}_{2}$ ): The presence of predictive validity requires identifying distinct features (here $F_{1}$ and $F_{2}$ ), each of which specifically explained by distinct models $M_{1}$ and $M_{2}$. One must show that there exists a real world transition (such as medication use) that results in transition from feature $F_{1}$ to $F_{2}$, and that this can be adequately modeled by a transformation of model $M_{1}$ into $M_{2}$. Face Validity (Panels $\mathrm{B}_{1}, \mathrm{~B}_{2}, \mathrm{C}_{1}, \mathrm{C}_{2}$ ): To establish face validity, one must identify features that characterize a target condition (such as bipolar disorder; here Condition $A$ ), denoted $F_{1}^{A}, F_{2}^{A}, \ldots, F_{N}^{A}$. Ideally, features that characterize a relevant comparator, Condition $B$, should also be identified $\left(F_{1}^{B}, F_{2}^{B}, \ldots, F_{K}^{B}\right)$. If model $M$ has face validity, then it should be able to explain as many features of condition A as possible, while not explaining features of condition B. Finally, if model $M$ explains some feature $F_{1}$, then it should not explain mutually exclusive features $F_{2}$. Construct Validity (Panels $\mathrm{D}_{1}, \mathrm{D}_{2}$ ): To establish construct validity, one must identify the components of a natural system, such as a biochemical pathway or neural circuit, and establish that functioning of that system explains some feature(s) F. A model system has construct validity if it is specified at a level of abstraction such that individual components and interactions are homologous to those present in the natural system. 


\subsubsection{Application of the Validity Appraisal Guide}

Two authors (AN, SS) independently applied the VAG-CM to papers selected for full-text review. After independent appraisal, the checklist responses for each paper were combined and inter-rater reliability was computed using Cohen's $\kappa$. Our measurement and reporting of $\kappa$ served to index the difficulty of interpreting each study in the context of their application to BD. After inter-rater reliability analysis, we resolved inconsistencies and conflicts by group consensus, with the resulting data compiled into tabular format. Hierarchical clustering in the $\mathrm{R}$ programming language (v. 4.1.2) was performed on the consensus checklist data to evaluate the degree to which ratings were consistent across papers with similar models and designs.

\section{RESULTS}

A total of 128 titles were returned from the initial search and 20 additional papers were identified by searching reference lists. After title/abstract screening, 36 full-text papers remained, of which 26 met inclusion criteria. The remaining 10 papers did not propose generative computational models of $\mathrm{BD}$.

Initial independent review of papers yielded a mean Cohen's $\kappa$ of 0.55 , with a $95 \%$ confidence interval (CI) of $(0.45,0.64)$. Cohen's $\kappa$ values for individual papers are plotted in Figure C.5. A summary of VAG-CM ratings is shown in Table 1, with full results shown in Table D.1.

Hierarchical clustering of VAG-CM data yielded three major groups of studies (Figure C.5), respectively summarized by the following attributes: theory-driven models with predictive validity, theory-driven models lacking face, predictive, and construct validity, and data-driven models with face validity. The specific cluster assignment is less important than identification of the fact that studies from the same groups, or employing similar approaches, tend to be closely co-located on the dendrogram, indicating that a given study design or model resulted in consistent evaluations on the VAG-CM. For instance, Ortiz et al. $[28,35]$ both employed autoregressive (AR) models, as did Bonsall et al. [21] and Moore et al. [39, 40]. Bayani et al. [31], Doho et al. [37], Nobukawa et al. [38] all employed the same model architecture, as did several other closely co-located studies $[16,19,24]$. Three notable exceptions formed their own cluster of studies, owing to demonstration of some degree of predictive validity $[11,20,25]$.

\subsection{Primarily Data-Driven Models with Face Valid- ity}

Many studies employed AR models which assume that the value of mood (or energy, activation, etc.) at time $t$ is a linear function of its own values at the previous $1 \leq k \leq t$ recordings. Although not constructed to be explicitly homologous with any physiological or psychological systems, AR models offer statistically rigorous explanations of time series data in BD $[21,28,35,39,40]$. Most of these models showed that selfratings from $\mathrm{BD}$ are often well predicted by self-ratings at the previous time step (i.e. the AR[1] model) [27, 39] (Cohen's $\kappa=0.8$ and 0.54 , respectively), although this is likely also the case for healthy controls [28] $(\kappa=0.94)$ and unaffected first-degree relatives of BD patients [35] $(\kappa=0.88)$.

Other studies that demonstrated face validity used models based on coupled stochastic differential equations which do not explicitly assume that mood is linearly dependent on its past values. Using self-report data collected every two months from $178 \mathrm{BD}$ patients, Cochran et al. [34] $(\kappa=0.42)$ evaluated several predictions made by existing theoretical models of mood dynamics in BD. The first assumption they tested was that mood fluctuations are inherently rhythmic [18-20, 22, 23, 27], which could not be conclusively proven or disproven based on their data. The second assumption was that mood states in BD such as mania, depression, and euthymia are themselves stable states (attractors) in which patients' moods become stuck, which would be evinced by multiple modes in a distribution of mood ratings [20, 23, 25]. Here, their data supported a unimodal hypothesis, whereby mood episodes are likely best captured as extremes of mood fluctuation. The third tested assumption was that mood is a one-dimensional construct with depression and mania at opposite poles $[18,19,21,25]$. They found this hypothesis to be inconsistent with their data based on the existence of mixed episodes. These findings led them to develop the affective instability model which assumes that mania and depression are governed separately, but may be positively or negatively correlated. Their model may thus account for mixed episodes (positive correlation of mania and depression) or shifts between exclusively manic or depressed states (negative correlation of mania and depression). Their model assumes that BD does not have intrinsic periodicity. Rather, they predict that individuals can end up in pathological mood states for two reasons: (A) a patient's baseline mood is already close to pathological levels, meaning small perturbations can bring mood into pathological states, or (B) mood is particularly sensitive and reaches pathological levels easily.

Two studies employed Markov Chain models with finite states (either observable or latent). One was able to identify discrete episodes (euthymia, depression, and mixed states) in longitudinal continuous self-report data [36]. The other showed that the pattern of mood state transition probabilities across subjects may relate to suicide attempts, as well as disability and disease chronicity [29].

One study used a van der Pol oscillator [41, 42] model to directly explain the findings of Gottschalk, Bauer, and Whybrow [43]: that mood dynamics in BD are governed by a system that is more deterministic (less random) than that observed in healthy controls [17] $(\kappa=0.38)$. Their model suggests that biological systems governing mood in BD either lack internal randomness, or are resistant to external sources of noise.

One study hypothesized that mood trajectories in BD are generated by two separate oscillating systems that can be coupled with varying degrees of strength [27] $(\kappa=0.54)$. They fit their mathematical model to QIDS-SR data collected from 25 patients with $\mathrm{BD}$, ultimately predicting that if a system of neural oscillators underlying mood dynamics can be identified, they will oscillate independently of each other in the majority of $\mathrm{BD}$ patients. 


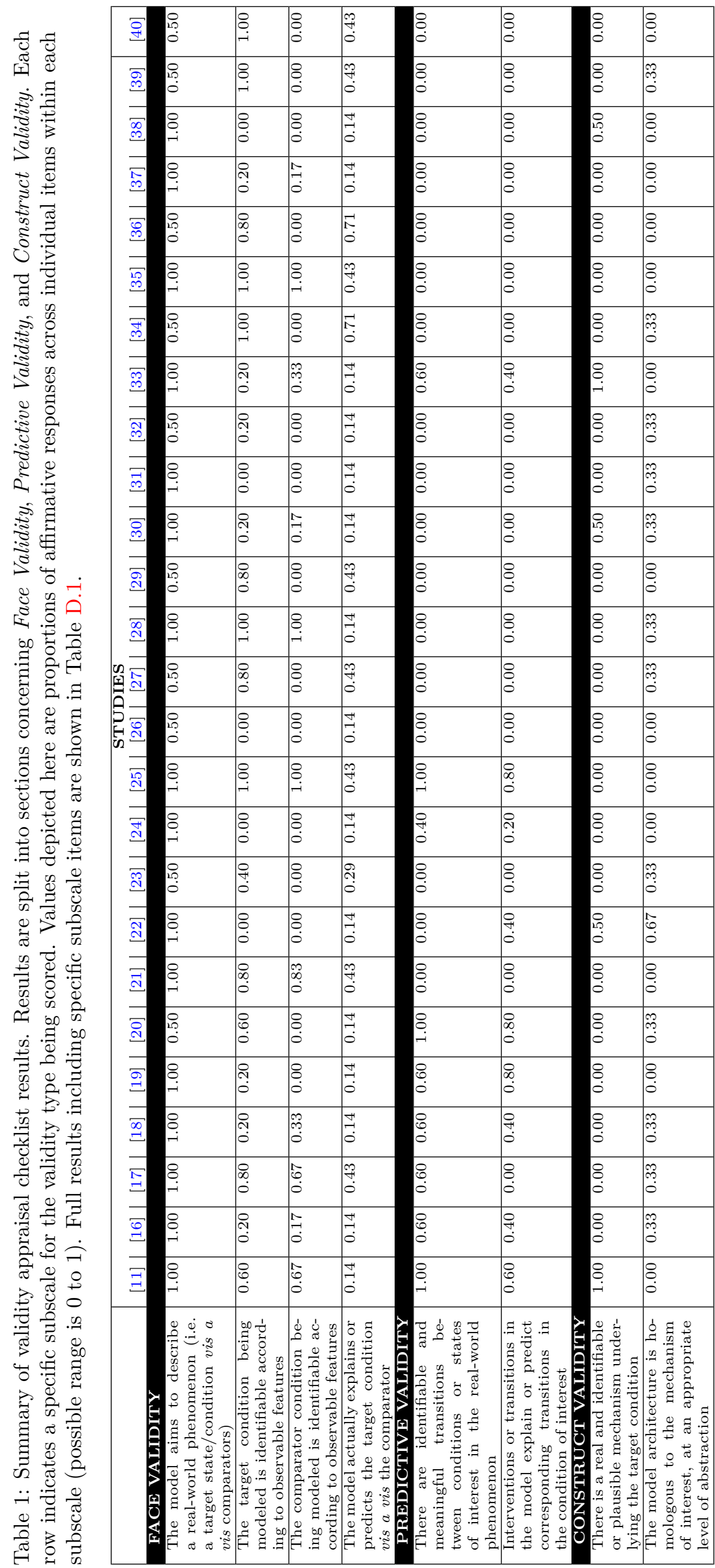




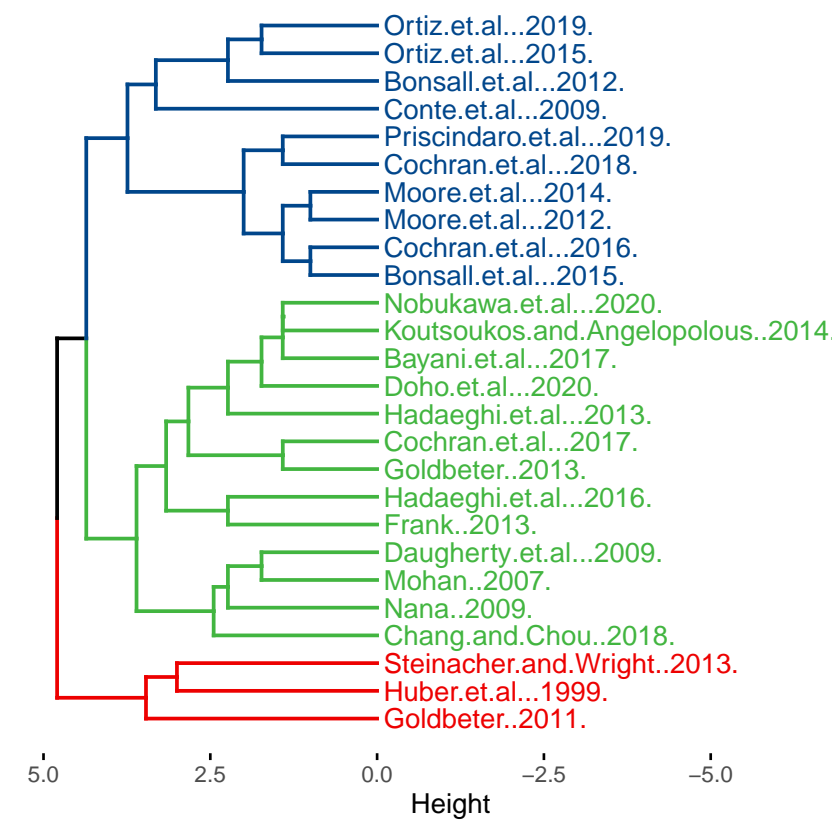

(a) Dendrogram showing clustering of papers.

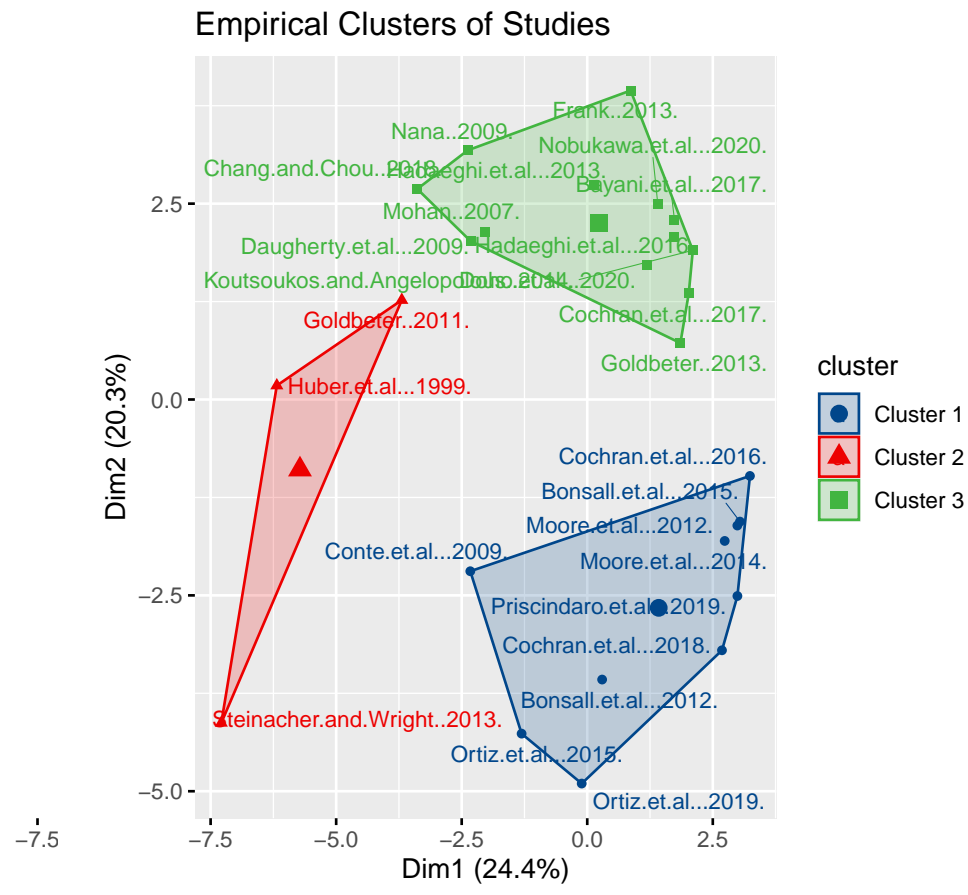

(b) Clusters along the first two principal components.

Figure 2: Results of paper clustering according to results on the validity appraisal guide. Cluster 1 corresponds to largely data-driven models that showed strong face validity. Cluster 2 corresponds to studies presenting theory-driven models with predictive validity. Cluster 3 corresponds to studies presenting theory-driven models that largely lacked face, predictive, and construct validity.

\subsection{Theory-Driven Models with Predictive Validity}

Huber et al. [11] $(\kappa=0.35)$ adapted the model of a single spiking neuron to model mood episodes as binary events (see Appendix A for details), with the aim of modeling phases of $\mathrm{BD}$ progression and the kindling phenomenon. Their model predicts that early in the disease course, individuals with more life events should show greater aperiodicity in episodes (compared to those with few life events). As time progresses, all individuals should enter a stable periodic regime with relative insensitivity to life events, followed by multistable and chaotic regimes late in the illness.

Goldbeter [20, 23] ( $\kappa=0.65$ and 0.43 , respectively) proposed a set of models assuming that mania and depression were independent attractor states, between which an individual's mood could oscillate in a bistable region. Goldbeter [20] predicted that if a depressed patient were given a sufficient antidepressant dose, the system governing mood oscillations would enter the bistable region, in which manic switch could occur. The Goldbeter [20,23] model makes a prediction that could be experimentally scrutinized: that the risk of antidepressant-induced manic switch in bipolar depression should be dose dependent at the individual level.

Steinacher and Wright [25] $(\kappa=0.68)$ presented the only model that could capture inter-episodic euthymia along with mania and depression. They modeled behavioral activation as a scalar value $X$ whose change over time is proportional to $X^{n}$, where $n \geq 0$ is a parameter governing "self-excitation" of the behavioral activation system (BAS). When $n=1$, the contribution of $X$ to self-excitation is linear. When $n>1$, a phenomenon of synergistic activation will be observed, where each additional unit increase of $X$ will disproportionately increase the amount of self-excitation (e.g. activation begets more activation). Finally, when $n<1$, each unit of increase in $x$ produces marginally less self-excitation.

The Steinacher and Wright [25] model predicts that hypersensitivity of the BAS, indexed by $n$, may relate to the propensity for mood episodes [44]. The authors compared BAS recovery time in their model to published data in which BAS recovery time is prolonged in $\mathrm{BD}$ patients with more mood episodes [45]. Thus, Steinacher and Wright [25] implicitly predict that their models, if fit to longitudinal BAS recordings from BD patients, should recover values of $n$ in proportion to patients' respective number of mood episodes.

The studies described in this section shared the common strength of building models to explain features of BD that were cited from relevant literature. However, a common limitation is that no model was designed with an architecture based on biological or psychological substrates of $\mathrm{BD}$, thus limiting construct validity.

\subsection{Theory-Driven Models Lacking Face, Predictive, and Construct Validity}

Several models attempted to capture mood oscillations [16, $18,19,22,24,26]$ or circadian variations of activity in BD $[24,31,37,38]$. We will not review each study in detail, but rather discuss common features that limited achievement of 
candidacy for face, predictive, or construct validity. Most prominently, the majority of studies failed to clearly define features that would be representative of the target condition (usually BD) or comparators being modeled. Often, mood oscillations were taken as the sine qua non of $\mathrm{BD}$, without clear operationalization or differentiation from patterns of mood fluctuation in other conditions or healthy individuals.

The study by Chang and Chou [33] $(\kappa=0.38)$ may be a notable exception to the other papers in this cluster. They leveraged the reinforcement learning framework to hypothesize that mood shifts are based on differences between realized and expected value. Including this framework provided some measure of potential construct validity, although not all model elements could be linked to underlying physiological or psychological substrates.

Chang and Chou [33] argued that mood fluctuations in BD arise from a hypersensitivity to noise, insofar as this noise generates prediction errors (differences between expectation and reality). They predict that self-sustained mood oscillations are induced when mood sensitivity exceeds a critical threshold, at which point mood is excessively sensitive to random noise in the environment. This model implicitly predicts that, in patients with presumed unipolar depression, the rate of antidepressant-induced manic switch should be proportional to the increase in mood sensitivity experienced during the antidepressant treatment.

\section{DISCUSSION}

We have presented a critical review of dynamical systems models of BD using a novel validity appraisal guide for computational models inspired by the animal model literature [12]. Using this approach, we were able to identify three clusters of studies: those employing data-driven methods with face validity, those employing theory-driven methods with higher predictive validity, and studies lacking in all forms of validity Taken together, these findings highlight the need to combine data-driven and theory-driven approaches for development of dynamical systems models of BD. These studies should (A) use data-driven approaches to identify critical features of BD and relevant comparators, and then (B) develop generative models to explain those features. Preferably, the latter generative models should be developed with some homology to physiological or psychological systems involved in BD. In the remainder of this discussion, we present a roadmap for this line of research.

The first key element in designing a dynamical systems model for BD is clearly identifying the condition and comparators of interest, and the features that define them. For instance, Ortiz et al. [35] identified BD, unaffected relatives, and the healthy control state as target and comparator states, respectively. In comparison, many studies unfortunately did not include comparator states, which precludes any assessment of their discriminant validity.

Studies must also clearly define a priori the features which are deemed central to explanation of target and comparator conditions. The identification of multiple features would be ideal since this contributes further to convergent validity of an explanatory model. Studies employing AR models were generally excellent in this respect, using multiple descriptive statistics to identify features of BD time series. For example, Ortiz et al. [28] showed that mood, anxiety, and energy selfratings during euthymia are Gaussian distributed, and that mood and anxiety are negatively cross-correlated (increases in mood are associated with reduction of anxiety). Using similar approaches, Ortiz et al. [35] found lower multiscale entropy levels in mood and energy time series from euthymic BD patients and unaffected first-degree relatives, compared to healthy controls. Interestingly, time series entropy in mood self-reports has been shown to increase in the 60 days prior to a manic or depressive episode, compared to the 60 days prior to a month of euthymia Glenn et al. [46]. Future computational modeling research should attempt to build mechanistic generative models of mood dynamics that can explain these features.

After defining target and comparator conditions, along with central features to be explained, future studies must ensure that proposed models are appropriately fit to these data. The optimal approach would be to fit the generative models to raw data, as was done by several previous authors [21, 27, 28, 34$36,39,40]$. However, we do not believe this is always necessary. In some cases, it may suffice to show that a model generates behavior corresponding qualitatively or ordinally to effects observed in humans. For instance, Steinacher and Wright [25] showed that increasing the BAS self-activation parameter $n$ resulted in prolonged behavioral activation recovery times after perturbation of their model, similar to that observed in BD patients with many prior episodes. Although not fit statistically to raw data, their model explained a clear effect from cited literature, which is nonetheless useful.

Future studies must develop computational models whose architectures are constrained by known biological or psychological features of BD. For example, Frank [22] presented a model with components that are homologous with systems of second messengers known to be involved in BD [47-49]. Although that particular study lacked face validity, it identified a potential biological dynamical system that could explain time series data in BD. Another dynamical system, the circadian pacemaker, exhibits disruptions in BD patients [50] and warrants further modeling, particularly as lithium response may be partially mediated by chronobiological mechanisms [51].

Finally, dynamical systems models of BD should strive toward achievement of predictive validity. As an example, one may consider modeling the results of Glenn et al. [46], who consider the change in affective dynamics occurring in the lead-up to mood episodes. One possible candidate for such a model is that of Chang and Chou [33], which predicts that the increased entropy in the 60 days preceding a mood episode would be related to a heightened mood sensitivity. Since the Chang and Chou [33] model is based on reinforcement learning, one could plausibly test this hypothesis in the laboratory $[52,53]$. Its basis in reinforcement learning also offers potential neurobiological connections and a prospect of construct validity [54-56].

A strength of the present study was the guided evaluation 
of studies by multiple raters using an a priori defined checklist based on accepted criteria in the animal modeling literature [12]. Although we applied the VAG-CM specifically to dynamical systems models of $\mathrm{BD}$, it would be of interest to evaluate its utility for model appraisal in the computational psychiatric neuroscience literature more broadly.

One limitation of our review is the inclusion of studies modeling different measures, collected at different durations and frequencies. To some degree this limits the ability to make direct comparisons of predictions across papers. Another limitation of our study is that some included papers were evaluated with low inter-rater reliability after initial screening. However, we believe this speaks to the need for a structured approach to evaluation, such as that provided by our appraisal guide. Fundamentally, the aims, design, and outcomes of studies with poor inter-rater reliability were difficult to appraise independently, which is in itself informative of the clarity and quality of those modeling studies.

In conclusion, by approaching our review of dynamical systems models of BD in a structured fashion (using the VAG$\mathrm{CM}$ ), we have identified a disconnect between the data-driven and theory-driven approaches used in the current BD modeling literature. We argue that taking a blended approach that combines strengths of both data-driven and theory-driven methods will ensure future models adequately explain behavior in a target condition, generate results that can be empirically verified, and provide mechanistic insights into BD through homology with biological and psychological systems. By developing biologically or psychologically plausible (i.e. homologous) models of the BD phenotype, we will step closer toward understanding how the brain generates this severe condition, and how its management can be improved.

\section{REFERENCES}

[1] K. S. Kendler, American Journal of Psychiatry 173, 771 (2016).

[2] K. S. Kendler, Psychological Medicine 47, 1013 (2017).

[3] J. Scott, G. Murray, C. Henry, G. Morken, E. Scott, J. Angst, K. R. Merikangas, and I. B. Hickie, JAMA Psychiatry 74, 189 (2017).

[4] I. Grande, M. Berk, B. Birmaher, and E. Vieta, The Lancet 6736, 1 (2015).

[5] S. Gershon, K. N. Chengappa, and G. S. Malhi, Bipolar Disorders 11, 34 (2009).

[6] R. Brady Jr, D. Öngür, and M. Keshavan, Harvard review of psychiatry 22, 23 (2014).

[7] A. Nunes, R. Ardau, A. Berghöfer, A. Bocchetta, C. Chillotti, V. Deiana, J. Garnham, E. Grof, T. Hajek, M. Manchia, B. Müller-Oerlinghausen, M. Pinna, C. Pisanu, C. O'Donovan, G. Severino, C. Slaney, A. Suwalska, P. Zvolsky, P. Cervantes, M. del Zompo, P. Grof, J. Rybakowski, L. Tondo, T. Trappenberg, and M. Alda, Acta Psychiatrica Scandinavica 141, 131 (2020).
[8] S. Lynch, Dynamical Systems with Applications Using Mathematica, 2nd ed. (Birkhauser, Boston, MA, 2017).

[9] S. H. Strogatz, Nonlinear dynamics and chaos: with applications to physics, biology, chemistry, and engineering, second edition ed. (Westview Press, a member of the Perseus Books Group, Boulder, CO, 2015) oCLC: ocn842877119.

[10] L. Rigoux, K. Stephan, K. Friston, and J. Daunizeau, NeuroImage 84, 971 (2014).

[11] M. T. Huber, H. A. Braun, and J. C. Krieg, Biological Psychiatry 46, 256 (1999).

[12] C. Belzung and M. Lemoine, Biology of Mood \& Anxiety Disorders 1, 9 (2011).

[13] S. Stern, A. Sarkar, D. Galor, T. Stern, A. Mei, Y. Stern, A. P. Mendes, L. Randolph-Moore, G. Rouleau, A. G. Bang, R. Santos, M. Alda, M. C. Marchetto, and F. H. Gage, Biological Psychiatry 88, 150 (2020).

[14] S. Becker and J. Lim, Journal of Cognitive Neuroscience 15, 821 (2003).

[15] M. Y. Yim, A. Hanuschkin, and J. Wolfart, Hippocampus 25, 297 (2015),_eprint: https://onlinelibrary.wiley.com/doi/pdf/10.1002/hipo.22373.

[16] M. A. Mohan, in Conference Proceedings - IEEE SOUTHEASTCON (2007) pp. 279-282.

[17] E. Conte, A. Federici, G. Pierri, L. Mendolicchio, and J. P. Zbilut, in Progress in Chaos and Complexity Research (Nova Research, New York, NY, 2009) pp. 25-44.

[18] D. Daugherty, T. Roque-Urrea, J. Urrea-Roque, J. Troyer, S. Wirkus, and M. A. Porter, Communications in Nonlinear Science and Numerical Simulation 14, 2897 (2009), arXiv:0311032 [nlin] .

[19] L. Nana, Communications in Nonlinear Science and Numerical Simulation 14, 351 (2009).

[20] A. Goldbeter, Progress in Biophysics and Molecular Biology 105, 119 (2011).

[21] M. B. Bonsall, S. M. A. Wallace-Hadrill, J. R. Geddes, G. M. Goodwin, and E. A. Holmes, Proceedings of the Royal Society B: Biological Sciences 279, 916 (2012), publisher: Royal Society.

[22] T. D. Frank, Communications in Nonlinear Science and Numerical Simulation 18, 2107 (2013).

[23] A. Goldbeter, Pharmacopsychiatry 46 Suppl 1, 44 (2013).

[24] F. Hadaeghi, M. R. Hashemi Golpayegani, and S. Gharibzadeh, Frontiers in Computational Neuroscience (2013), 10.3389/fncom.2013.00106.

[25] A. Steinacher and K. A. Wright, PLoS ONE 8 (2013), 10.1371/journal.pone.0063345. 
[26] E. Koutsoukos and E. Angelopoulos, International Journal of Bipolar Disorders 2, 1 (2014).

[27] M. B. Bonsall, J. R. Geddes, G. M. Goodwin, and E. A. Holmes, Journal of The Royal Society Interface 12, 20150670 (2015).

[28] A. Ortiz, K. Bradler, J. Garnham, C. Slaney, and M. Alda, Bipolar Disorders 17, 139 (2015).

[29] A. L. Cochran, M. G. McInnis, and D. B. Forger, Translational Psychiatry 6, e912 (2016).

[30] F. Hadaeghi, M. R. Hashemi Golpayegani, S. Jafari, and G. Murray, Australian and New Zealand Journal of Psychiatry 50, 783 (2016).

[31] A. Bayani, F. Hadaeghi, S. Jafari, and G. Murray, Chronobiology International 34, 235 (2017).

[32] A. L. Cochran, A. Schultz, M. G. McInnis, and D. B. Forger, in Computational Neurology and Psychiatry, Vol. 6, edited by P. Érdi, B. Sen Bhattacharya, and A. L. Cochran (Springer International Publishing, Cham, 2017) pp. 315-341, series Title: Springer Series in Bio/Neuroinformatics.

[33] S.-S. Chang and T. Chou, Computational Psychiatry 2, 205 (2018).

[34] A. L. Cochran, A. Schultz, M. G. McInnis, and D. B. Forger, Translational Psychiatry 8, 36 (2018).

[35] A. Ortiz, K. Bradler, J. Garnham, C. Slaney, S. McLean, and M. Alda, Journal of Affective Disorders 243, 274 (2019).

[36] J. J. Prisciandaro, B. K. Tolliver, and S. M. DeSantis, Psychological Medicine 49, 1102 (2019).

[37] H. Doho, S. Nobukawa, H. Nishimura, N. Wagatsuma, and T. Takahashi, Frontiers in Computational Neuroscience 14, 76 (2020).

[38] S. Nobukawa, H. Nishimura, H. Doho, and T. Takahashi, Frontiers in Applied Mathematics and Statistics 6, 53 (2020).

[39] P. J. Moore, M. A. Little, P. E. McSharry, J. R. Geddes, and G. M. Goodwin, IEEE Transactions on Biomedical Engineering 59, 2801 (2012).

[40] P. J. Moore, M. A. Little, P. E. McSharry, G. M. Goodwin, and J. R. Geddes, International Journal of Bipolar Disorders 2, 1 (2014).

[41] B. van der Pol, Radio Review (London) 1, 701 (1920).

[42] B. van der Pol, The London, Edinburgh, and Dublin Philosophical Magazine and Journal of Science 2, 978 (1926).

[43] A. Gottschalk, M. S. Bauer, and P. C. Whybrow, Archives of general psychiatry 52, 947 (1995).
[44] S. Urošević, L. Y. Abramson, E. Harmon-Jones, and L. B. Alloy, Clinical Psychology Review 28, 1188 (2008).

[45] K. A. Wright, D. Lam, and R. G. Brown, Journal of Abnormal Psychology 117, 838 (2008).

[46] T. Glenn, P. C. Whybrow, N. Rasgon, P. Grof, M. Alda, C. Baethge, and M. Bauer, Bipolar Disorders 8, 424 (2006).

[47] Y. Bezchlibnyk and L. T. Young, The Canadian Journal of Psychiatry 47, 135 (2002), publisher: SAGE Publications Inc.

[48] C. A. Zarate and H. K. Manji, CNS drugs 23, 569 (2009).

[49] A. Saxena, G. Scaini, D. Bavaresco, C. Leite, S. Valvassoria, A. Carvalho, and J. Quevedo, Molecular Neuropsychiatry 3, 108 (2017).

[50] R. Gonzalez, 75, e323, place: United States.

[51] H. K. Mishra, N. M. Ying, A. Luis, H. Wei, M. Nguyen, T. Nakhla, S. Vandenburgh, M. Alda, W. H. Berrettini, K. J. Brennand, J. R. Calabrese, W. H. Coryell, M. A. Frye, F. H. Gage, E. S. Gershon, M. G. McInnis, C. M. Nievergelt, J. I. Nurnberger, P. D. Shilling, K. J. Oedegaard, P. P. Zandi, The Pharmacogenomics of Bipolar Disorder Study, J. R. Kelsoe, D. K. Welsh, and M. J. McCarthy, Molecular Psychiatry 26, 3383 (2021).

[52] E. Eldar and Y. Niv, Nature Communications 6, 1275 (2015).

[53] E. Eldar, R. B. Rutledge, R. J. Dolan, and Y. Niv, Trends in Cognitive Sciences 20, 15 (2016).

[54] Y. Niv, Journal of Mathematical Psychology 53, 139 (2009).

[55] D. Lee, H. Seo, and M. W. Jung, Annual Review of Neuroscience 35, 287 (2012).

[56] J. P. O'Doherty, S. W. Lee, D. Mcnamee, J. P. O'Doherty, S. W. Lee, and D. Mcnamee, Current Opinion in Behavioral Sciences 1, 1 (2015).

\section{A OVERVIEW OF DYNAMICAL SYSTEMS}

In order to facilitate interpretation of the models uncovered by our review, this section first introduces some basic technical aspects of dynamical systems using simple examples. A dynamical system is a system whose state evolves over time in accordance to an "evolution rule", which can be mathematically described by sets of differential equations in the continuous setting, or difference equations in the discrete setting. Here, we provide a basic introduction to models based on differential equations.

Our first model is demonstrative of continuous dynamical systems that reach a stable equilibrium (a stable state or fixed point). These models may have one or more stable states, but are characterized by the system converging to, and remaining 
at, these points or limit sets until perturbed. The specific stable state to which a system arrives may depend on several factors, including the starting point. Example 1 describes a simple dynamical system modelling growth of a population under (A) space constraints and (B) predation, in which the population size reaches a stable equilibrium.

Example 1 (A System with Stable States). A classical dynamical system describes the growth of a population whose size at time $0 \leq t$ is denoted $x(t)$ or simply $x$ for parsimony. The rate of change in $x$, denoted $\dot{x}$ (alternatively $x^{\prime}(t)$ or $\mathrm{d} x / \mathrm{d} t$ ) is

$$
\dot{x}=a\left(1-\frac{x}{x_{\max }}\right) x,
$$

which is known as the logistic equation. Here, coefficient $x_{\max }$ denotes the maximum possible population size, and $a$ is a scalar value identifying the growth rate. If $a>1$, then the population will grow over time, whereas it will shrink if $a<1$. The term $\left(1-x / x_{\max }\right)$ indicates the proportion of population capacity still available for reproduction. As $x$ approaches $x_{\max }$, the value of $\left(1-x / x_{\max }\right)$ approaches 0 and reproduction stops.

Some populations are also subject to predation. This can be incorporated into Equation 1 by adding a predation function $b x^{2} /\left(c^{2}+x^{2}\right)$, where $b>0$ is a constant representing the rate of predation, and $c>0$ represents the size of the population at which $50 \%$ of the maximum predation rate is reached (note that this is equivalent to the biochemical Hill function with exponent $n=2)$. Equation 1 thus becomes

$$
\dot{x}=a\left(1-\frac{x}{x_{\max }}\right) x-b \frac{x^{2}}{c^{2}+x^{2}} .
$$

Figure A.1 is a stream plot showing the trajectories of population size (as modelled by Equation 2) across time. For initial population sizes greater than 0, the population will converge on a stable equilibrium value. The rate at which the population approaches that fixed point is dependent on the initial population size.

Instead of demonstrating stable fixed points, some systems show solutions that converge to limit cycle attractors, resulting in periodic oscillations. One such class of systems that will be encountered across many studies in our review [11, 16$19,24,27]$ is the relaxation oscillator ( $\mathrm{RO})$, most notably the van der Pol oscillator (VPO) [41, 42]. Although population dynamics models, including those adapted for analysis of biochemical kinetics can also produce oscillatory behaviour [22, 25], Example 2 will introduce the VPO.

Example 2 (A System with Periodic Oscillations). A van der Pol oscillator [41, 42] is a one-dimensional system with state $x(t)$ (here simply $x$ ) is defined according to the following second order ordinary differential equation:

$$
\ddot{x}=\mu\left(1-x^{2}\right) \dot{x}-x,
$$

where "second order" refers to the fact that these dynamics are expressed in terms of the second derivative of $x$ with respect to time: $\ddot{x}=x^{\prime \prime}(t)=\mathrm{d}^{2} x / \mathrm{d} t^{2}$. The parameter $\mu$ is a scalar value representing the amount of damping or nonlinearity. Figure A.2 shows the VPO behaviour across different values of $\mu$.

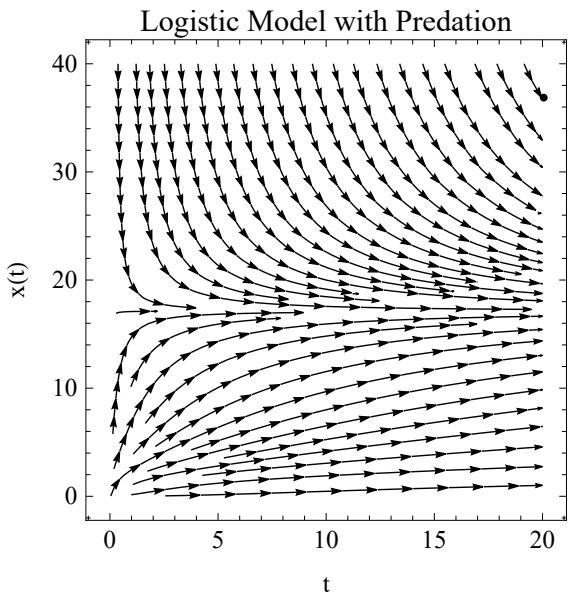

Figure A.1: Illustration of the different time-courses of growth of a population subject to predation, as expressed by the differential equation $\dot{x}=a x\left(1-x / x_{\max }\right)-b x^{2} /\left(c^{2}+x^{2}\right)$, with parameters $a=2, x_{\max }=20, b=5, c=2$. Each curve represents the trajectory of a population given initial value $x(0)=x_{0}$. Arrows denote the direction of time.

When $\mu<0$, the system will show a progressive damping of oscillations until $x$ approaches 0 . When $\mu=0$, the system shows simple harmonic motion. When $\mu>0$, we observe limit cycles (stable periodic trajectories to which the system will converge from multiple different starting points).

Figure A.2 also demonstrates the phenomenon of a "relaxation oscillation," whereby the system alternates between slow and rapid movements (depicted here when $\mu=7$ ).

One can appreciate why RO models might be proposed for BD mood dynamics. Superficially, BD consists of episodes in which an individual persists for some time, followed ostensibly by transition into a different state of relative stability. However, natural phenomena rarely proceed with such regular periodicity. Rather, naturally oscillating systems are often characterized by behaviour that appears almost random. Yet, seemingly random behaviour can emerge from a totally deterministic system in a phenomenon known as chaotic dynamics.

Example 3 (A Chaotic Continuous Dynamical System). Huber et al. [11] sought to model episodes of mania and depression as discrete (on/off) events, since their primary concern was the rhythmicity of episodes and inter-episode timing. This was implemented using a model of neuronal spiking illustrated graphically in Figure A.3, and characterized by the following ordinary differential equation,

$$
\tau_{x} \dot{x}=-x-\sum_{i \in \mathcal{A}} A_{i}^{v} w_{i}\left(x-x_{i}\right)+S+W
$$

where $x$ is analogous to a neuronal membrane potential, $\tau_{x}$ is a relaxation time constant, $S$ is a constant external input, and $W$ is Gaussian noise. The set $\mathcal{A}=\{$ he, hi, le, li $\}$ identifies different excitatory and inhibitory elements of the system. That is, the system contains high-threshold excitatory and inhibitory elements $A_{\mathrm{he}}$ and $A_{\mathrm{hi}}$, as well as low-threshold excitatory and inhibitory elements $A_{\mathrm{le}}$ and $A_{\mathrm{li}}$, respectively. These are 

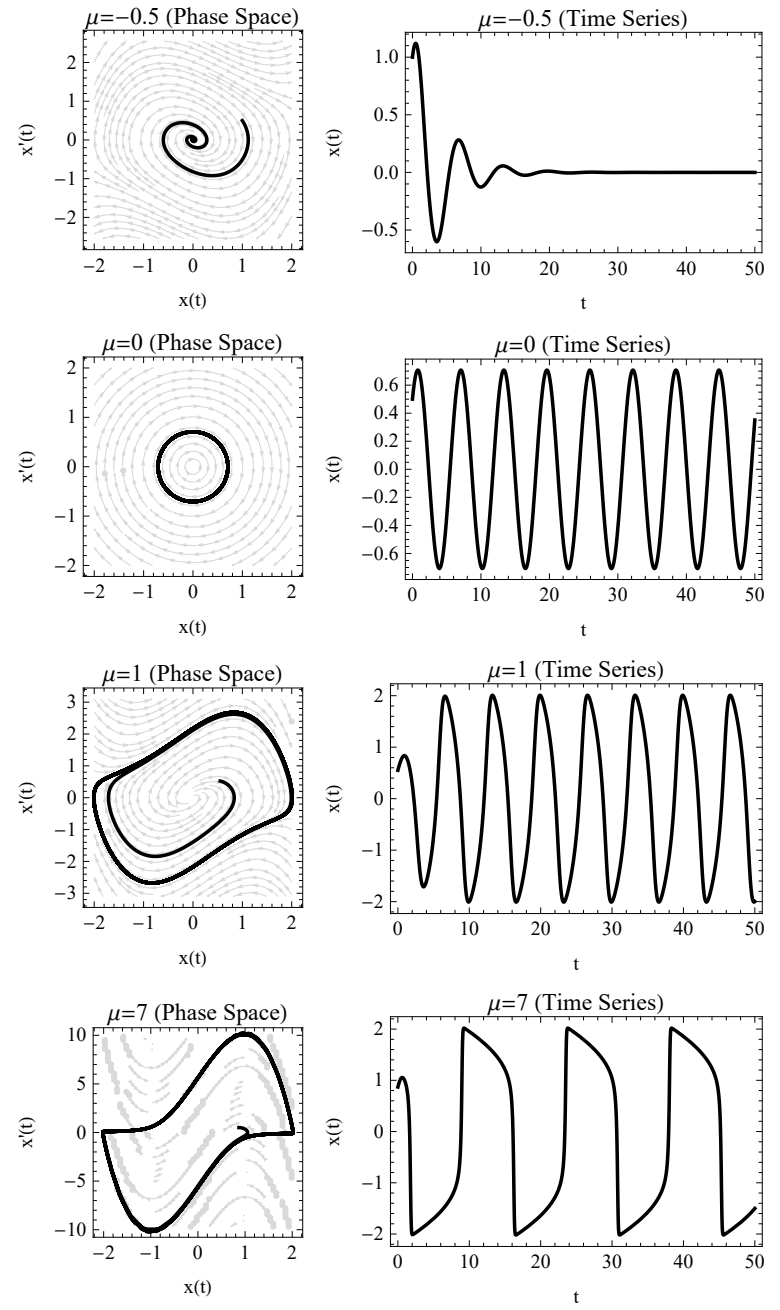

Figure A.2: The van der Pol oscillator (VPO) [41, 42] across values of the nonlinearity/damping parameter $\mu$. Each row of plots illustrates a VPO for different values of $\mu$. The left and right columns show the phase space portraits, and corresponding time series, respectively.

coupled to the state variable $x$ according to "synaptic weights" $w_{i} \in\left\{w_{\mathrm{he}}, w_{\mathrm{hi}}, w_{\mathrm{le}}, w_{\mathrm{li}}\right\}$ and activation threshold constants $x_{i} \in\left\{x_{\mathrm{he}}, x_{\mathrm{hi}}, x_{\mathrm{le}}, x_{\mathrm{li}}\right\}$. Huber et al. [11] set the exponent $v$ to 2 for $A_{\text {he }}$, and to 1 otherwise.

The system simulated by Huber et al. [11] can be conceptualized as a mixture of excitatory/inhibitory oscillating subsystems, whose states $A_{i} \in\left\{A_{\mathrm{he}}, A_{\mathrm{hi}}, A_{\mathrm{le}}, A_{\mathrm{li}}\right\}$ themselves evolve over time according to the following differential equation:

$$
\tau_{i} \dot{A}_{i}=f_{i}(x)-A_{i} .
$$

Here, $\tau_{i}$ is a relaxation time constant, and

$$
f_{i}(x)=\frac{1}{1+\exp \left\{\beta_{i}\left(x-x_{i, 0.5}\right)\right\}}
$$

is a sigmoid with slope $\beta_{i}$ and half-activation constant $x_{i, 0.5}$.

At different levels of deterministic input (S), this system is capable of simulating fixed-point or steady state dynamics, as well as periodic oscillations, limit cycles, and chaotic behaviour. Figure A.4 demonstrates this behaviour. With $S \approx 10$ or below,

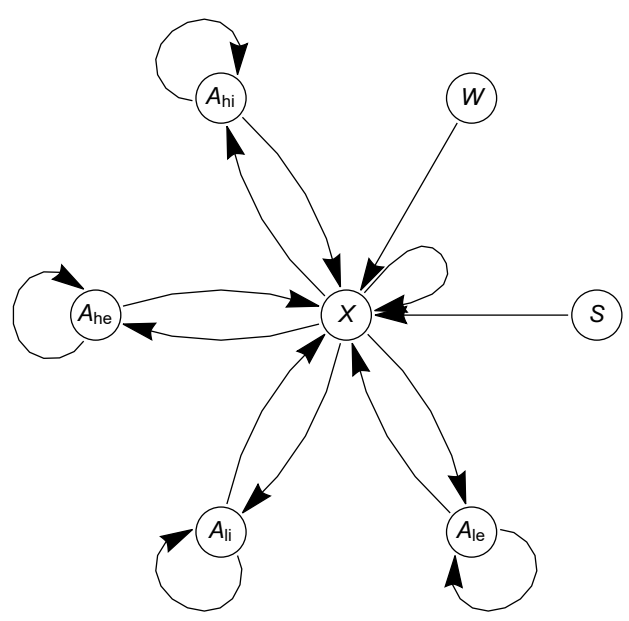

Figure A.3: Graphical illustration of the Huber et al. [11] model.

the system undergoes dampened oscillations to a limiting fixed point. At $S \approx 50$, we observe regular periodic spiking, and periodic bursts at $S \approx 100$. At levels of $S$ between approximately 175 and 325, we observe chaotic inter-spike intervals (see Huber et al. [11] for further detail through bifurcation plots).

In this section, we have introduced simple examples of continuous dynamical systems capable of generating multiple forms of behaviour, including fixed-points, stable oscillations and limit cycles, as well as chaotic dynamics. Two of these models, the RO (Example 2) and neuronal model (Example 3 ), are directly implemented in studies that will be discussed in Sections 3 and 4.

\section{B VALIDITY APPRAISAL GUIDE FOR COMPUTATIONAL MODELS}

\section{B.1 Subscale 1: Face Validity}

Definition: The degree to which the model exhibits a range of behaviours similar to that of the condition of interest In other words, how good is the model at explaining behaviour in the target condition?

1. The model aims to describe a real-world phenomenon (i.e. a target state/condition vis a vis comparators)

Target condition defined clearly

Comparator(s) defined clearly

2. The target state/condition being modelled is identifiable according to observable features

\section{Features are explicitly defined}

Feature definitions are operationalized with concrete criteria or measurement scales

Relevance of features to condition of interest is stated (i.e. features actually present clinically) 

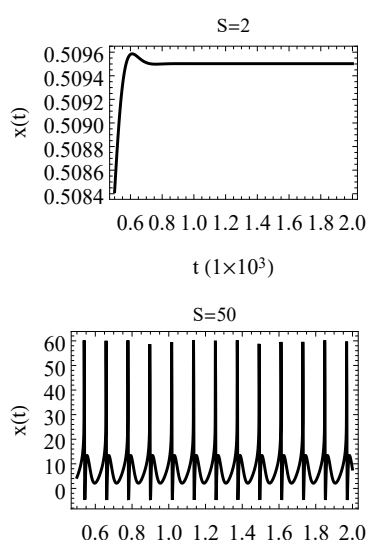

$\mathrm{t}\left(1 \times 10^{3}\right)$

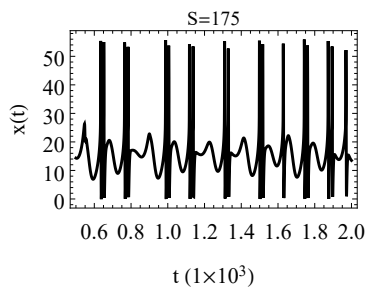

$\mathrm{S}=10$

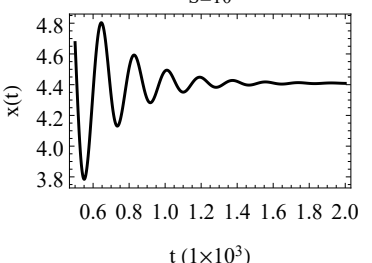

$\mathrm{S}=100$
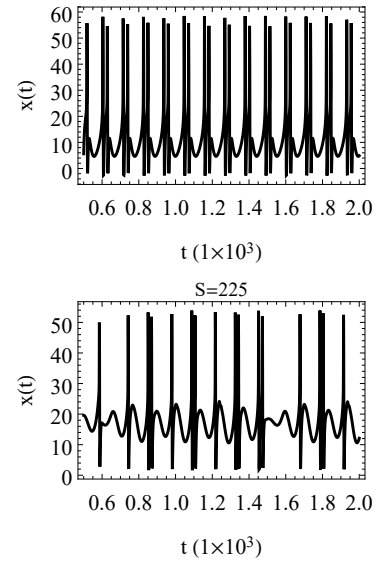

Figure A.4: Results of deterministc simulations of the Huber et al. [11] model at various levels of constant input $S$. This simulation was generated with the following parameters: $\tau_{x}=$ $10, \tau_{\mathrm{hi}}=2, \tau_{\mathrm{li}}=100, \tau_{\mathrm{le}}=10, x_{\mathrm{hi}}=-30, x_{\mathrm{he}}=110$, $x_{\mathrm{li}}=-30, x_{\mathrm{le}}=110, w_{\mathrm{hi}}=20, w_{\mathrm{he}}=15, w_{\mathrm{li}}=18, w_{\mathrm{le}}=3$, and initial conditions $x(0)=A_{i}(0)=0$ for all $i \in\{$ he, hi, le, li $\}$.

Features are identified based on cited empirical observations or experiments

Multiple features considered

3. The comparator state/condition being modelled is identifiable according to observable features

Features are explicitly defined

Feature definitions are operationalized

Relevance of features to condition of interest is stated (i.e. features actually present clinically)

Features are identified based on cited empirical observations or experiments

Multiple features considered

Relationship to features of target condition identified (e.g. mutual exclusivity, correlation, etc.)

4. The model actually explains/predicts the target condition vis a vis the comparator

All models included are generative (i.e. capable of simulating data)

Quality of model fit quantified statistically (with respect to empirical data)

Quality of model fits compared statistically between models (e.g. Bayesian Model Selection)

Model fit and comparisons were done with adequate statistical power
Proposed model is best fit for features of the target state/condition

Proposed model is not best fit for features of comparator (unless model is modified through intervention; see predictive validity)

Best fitting model does not generate additional features that are absent in the target state/condition

\section{B.2 Subscale 2: Predictive Validity}

Definition: The degree to which manipulations of the model predict the effects of real world interventions on the target condition of interest. In other words, do interventions on the model result in changes of its behaviour similar to those of an analogous intervention on the real-world target condition? For example, if model $M_{1}$ captures depression $(D)$, and model $M_{2}$ captures normal mood (euthymia, $E$ ), then some transformation $T\left(M_{1}, M_{2}\right)$ should be a good explanation for scenarios in which one observes $D \rightarrow E$.

1. There are identifiable and meaningful transitions between conditions/states of interest in the real-world phenomenon

States and transition are explicitly identified (e.g. depression $\rightarrow$ euthymia)

Nature by which transition occurs in reality is explicitly identified (e.g. medication intervention causing depression to transition into euthymia)

Nature of transition in reality is empirically or theoretically based, with appropriate citation

Relevance of transition for understanding the condition is explicitly defined (e.g. treatment effect)

Relevance of transition of understanding the condition has appropriate theoretical or empirical grounds, with included citations

2. Interventions/transitions in the model explain or predict corresponding transitions in the condition/state of interest

Distinct models are identified for corresponding states of interest

Individual models explain distinct states exclusively (see face validity)

The intervention resulting in transition between models is explicitly defined

Transformation of one model into the other corresponds to transformation between the target states OR Transformation of one model into the other predicts transformation between target states that can be verified empirically

Degree to which transformation between models corresponds to transformation between target states is quantified statistically, and deemed to be the best fit, at least in comparison to a "null" or random transformation OR Transformation of one model into the other predicts a transformation that should be observed in between the target states/conditions in the real-world. Prediction is quantitative, empirically verifiable, and includes degree of uncertainty 
Inter-Rater Reliability

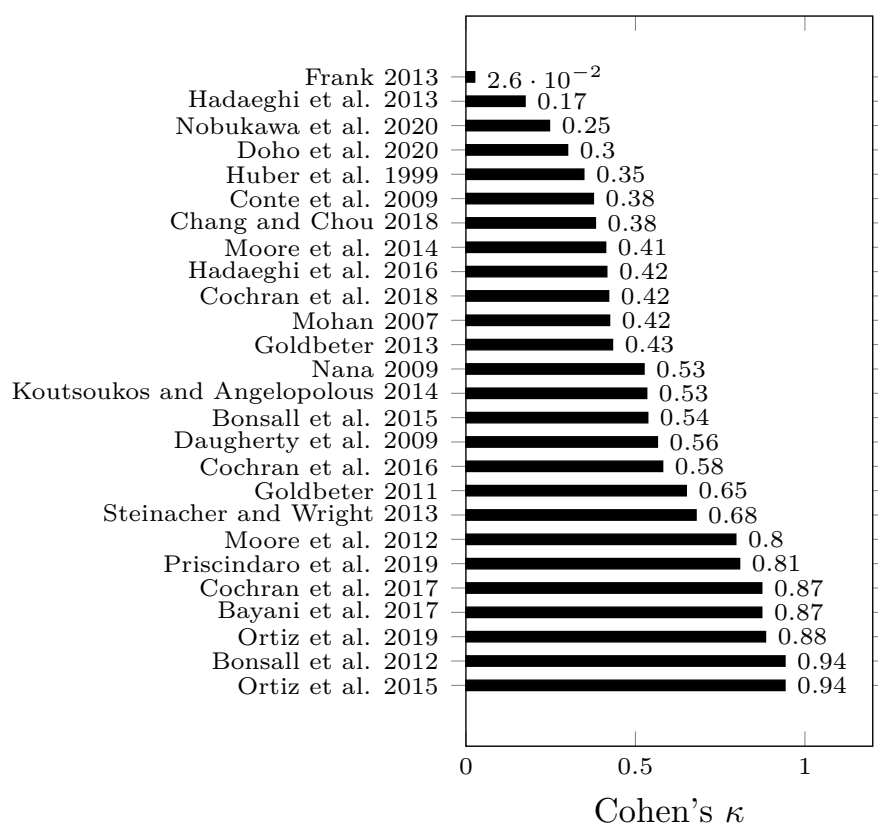

Figure C.5: Cohen's $\kappa$ statistics for each of the papers reviewed. Mean $\kappa$ was $0.55(95 \%$ CI $[0.45,0.64])$.

\section{B.3 Subscale 3: Construct Validity}

Definition: The degree of homology between the model and mechanisms that are empirically or theoretically deemed to underlie features of the target condition of interest.

1. There is a real and identifiable or plausible mechanism underlying the target condition/state

\section{Explicitly identified}

Empirically or theoretically shown to be involved/associated with target features being explained OR Model makes explicit testable predictions about the existence of this underlying mechanism

The model architecture is homologous to the mechanism of interest, at an appropriate level of abstraction

2. Model components are defined and linked to specific components of underlying mechanism

Functional arrangement/connections between model components defined and linked to underlying mechanisms

Multiple models were evaluated with competing mechanisms

\section{INTER-RATER RELIABILITY RESULTS}

Cohen's $\kappa$ for each individual paper reviewed is plotted in Figure C.5.

\section{VALIDITY APPRAISAL GUIDE RESULTS TABLE}

Complete results for raters' evaluations of papers using the Validity Appraisal Guide for Computational Models are shown in Table D.1. 


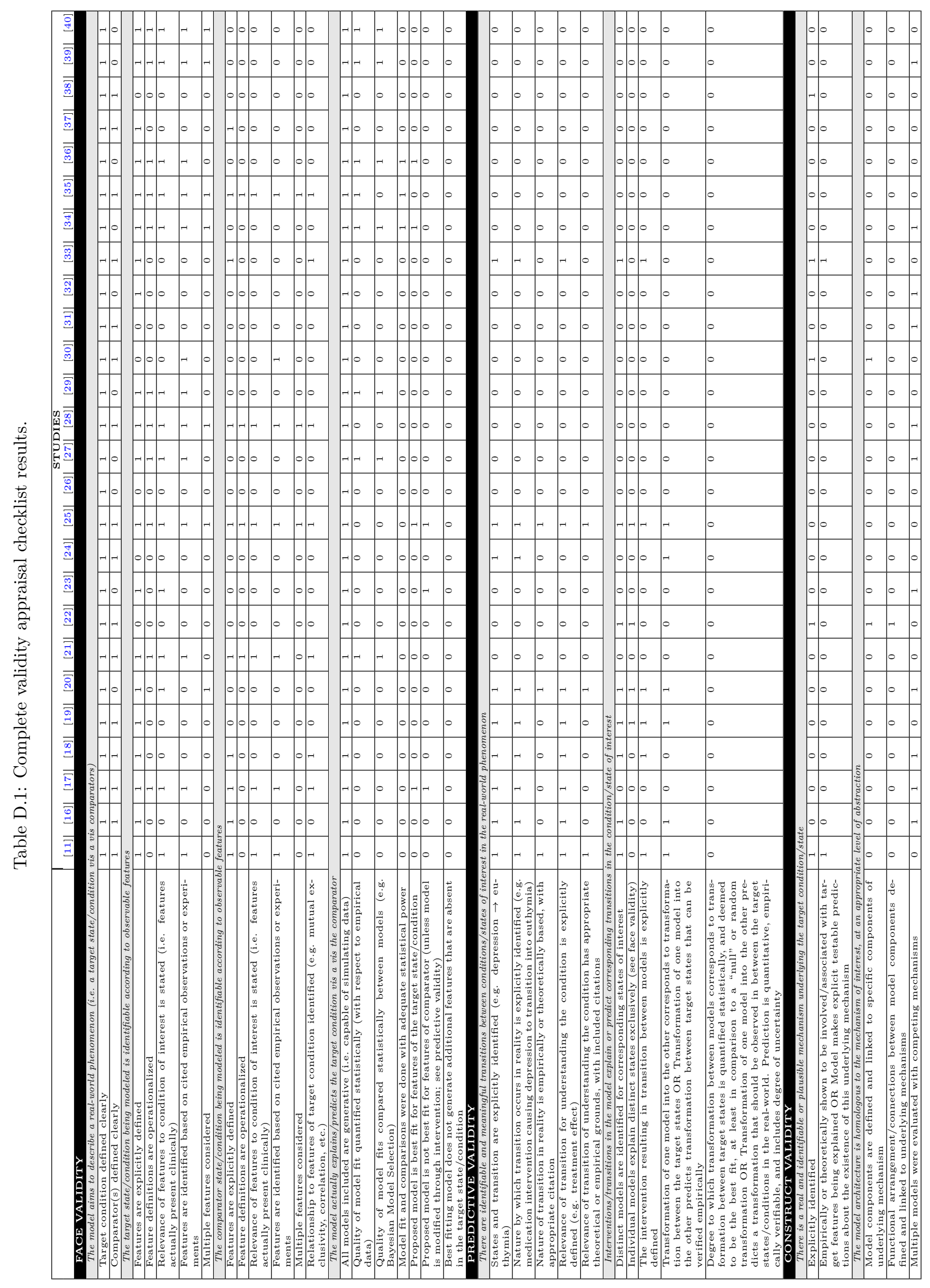

